\title{
Immunological functions of the gut in relation to nutritional state and mode of delivery of nutrients
}

\author{
Anne Ferguson
}

\begin{abstract}
Gut immunology encompasses the need for local cellular immunity and prevention of systemic immune reactions to dietary antigens. The relation between these factors and nutritional state or the presence of luminal nutrients in the enterally fed and parenterally fed is poorly defined. Most studies suggest that acquisition of lymphoid characteristics is independent of luminal nutrition and its responsiveness is related more to bacterial challenge. Protein malnutrition may impair immune responsiveness by moderating the generalised inflammatory response, rather than through reduced $T$ cell function and IgA synthesis. Predisposition to the development of gut hypersensitivity can be induced in animals by longterm feeding with elemental diets. The efficient absorption of these diets reduces the caecal microflora burden and together with changed gastric acid secretion and small bowel motility, may affect the composition of the gut flora. Changed luminal nutrition, enhanced tissue damage and inappropriately increased mucosal $T$ cell function may thus be related. The clinical effectiveness, however, of elemental diets in treatment of unresponsive coeliac disease is reassuring. To investigate intestinal immunity in humans, an approach based on whole gut lavage has been developed. Data from this noninvasive human technique will prove to be a useful means of assessing the effects of nutritional rehabilitation on mucosal immunity.
\end{abstract}

(Gut 1994; Supplement 1: S10-S12)

The key immune functions of the gut are the generation of secretory IgA antibody and of local cellular immunity (the second normally in response to certain infections) together with specific down regulation of systemic immunity to antigens presented through the gut (oral tolerance). Important in the maintenance of these functions are antigen handling and presentation; immunoregulatory factors in the microenvironment of the gut; and the presence of cells and molecules expressing specific and non-specific immune responses.

These concepts have been derived from work in experimental animals. Clinical studies of human intestinal immunity have, until recently, been greatly restricted by problems of access to intestinal secretions and mucosal biopsies, but new techniques are now being devised to overcome these problems.
Important questions to be answered with respect to enteral nutrition and gut immunity include: (a) are immune cells of the gut mucosa normal in numbers and properties when luminal nutrition is absent, or is presented in unusual physiochemical form? (b) how does the nutrition state in general affect gut immunity? Does provision of luminal nutrition in a normal or unusual form change the function of immune cells? This could be either useful or detrimental; (c) we already know that immune reactions in the gut can cause tissue damage, affect epithelial cell kinetics, and lead to malabsorption (hypersensitivity). Are these responses affected in any way by the provision of enteral nutrition?

The answers to these questions cannot yet be provided for humans, but the results of some of our animal experiments may be relevant, and can certainly act as a focus for discussion.

Luminal nutrition and immune cells of the gut mucosa

In the 1970 s I described a simple method for producing antigen free segments of small intestine or colon in rodents. ${ }^{1}$ Fragments of fetal small intestine were implanted under the kidney capsules of syngeneic adult recipients. In the third week after implantation these developed characteristics of adult rodent intestine. ${ }^{2} 3$ Peyer's patches, lamina propria, and the intraepithelial sites were populated by lymphocytes, although fewer in number than in normally sited gut and without germinal centres. Cell traffic studies showed normal migration of $\mathrm{T}$ and $\mathrm{B}$ immunoblasts and the retention of these cells in the tissues; their maturation and differentiation within these grafts was normal. ${ }^{4}$ We also showed that secretion of $\operatorname{IgA}$ occurred into the lumen of antigen free grafts. ${ }^{5}$

Thus, neither food nor intestinal bacterial flora is necessary to permit the gut to acquire its innate lymphoid constituents. Furthermore, in experiments with allografts of intestine we showed that inflammatory cells, and $\mathrm{T}$ cells capable of mounting a delayed type hypersensitivity (DTH) reaction, were recruited into tissues of these grafts. ${ }^{6}$ Experiments in animals with an expanded population of mucosal mast cells (induced by a gut parasite) showed that mast cell infiltration of grafts could also occur. ${ }^{7}$

This work, and a wealth of other data, highlights that lymphoid tissues are intrinsic constituents of the gut mucosa. Related studies also show that the intestinal bacterial flora is much more important than dietary factors in expanding the size of the gut associated lymphoid population. 


\section{Immune functions of the gut and nutritional state}

The microenvironment of the gut is critical in relation to induction of specific immune responses at the first encounter with fed antigen. Important factors include dose of antigen, its physicochemico properties, the route of administration, and other signals received by reacting cells when antigen is presented. On the other hand expression of the immune response occurs in a previously immunised host; the nature and effects of this expression will vary greatly depending on the cells and immunoglobulin classes participating, the site of the reaction, and the non-specific cells and molecules that are recruited to the site of the reaction. It is important to emphasise that expression of immunity may be entirely harmless, may confer protection for example by toxin neutralisation or bacterial killing, or may be adverse (allergy or hypersensitivity).

Theoretically there are many ways in which nutrition could affect gut immune state either as part of a general effect of nutritional state on the host, or in relation to the provision of certain nutrients in the microenvironment of the epithelial and subepithelium tissues. There are many possible ways in which nutrition might operate, for example on non-specific immunity; on the magnitude of the appropriate IgA response, or the induction of a potentially harmful immature response entailing IgM; on the induction of oral tolerance; in producing deficiency or inappropriately up regulating $T$ cell functions. Nutritional state will affect the capacity of lymphoid cells to generate cytokine signals and will also influence the capacity of epithelial, lymphoid, and other cell types to respond to such signals.

I have no data on the effects of nutritional state on antibody responses although there are several papers in published works, mainly on work in animals, suggesting that IgA deficiency is produced by protein malnutrition. A series of experiments, however, which we carried out in protein malnourished mice, mainly studying $\mathrm{T}$ cell immunity and tolerance, are of interest and illustrate the complexity of the situation and the need to use ingenuity and take plenty of time to follow through the necessary experiments.

The thymus dependent ( $T$ dependent) limb of the immune response in protein malnourished mice was examined by using several tests of $T$ cell function, both in vivo within the intact animal, and after removal of lymphocytes from the protein deprived host. ${ }^{8}$ The capacity to provide help for IgG antibody responses and the DTH response to antigen were diminished after short term (three weeks) deprivation. Both these responses, however, were normal in mice maintained on a protein deficient diet for 11 weeks. The depressed DTH responses of protein deprived mice were a result, at least in part, of a failure to mount the inflammatory phase of the response. Finally, using a graft $v$ host reaction (GvHR) as an index of $T$ lymphocyte function, we found that spleen cells from malnourished donors could induce the splenic and intestinal changes associated with a
GvHR in unirradiated $F_{1}$ animals. Overall, these results suggested that $T$ cell function is not irreversibly damaged by protein deprivation. We proposed that cell mediated immune responses are influenced indirectly by the restrictive environment, which interferes with cell migration, and by the impaired capacity of protein deprived animals to mount non-specific inflammatory reactions.

We then examined the effects of protein deprivation on the induction of oral tolerance for systemic antibody and DTH responses to the protein antigen ovalbumin. ${ }^{9}$ Mice were fed 4 or $24 \%$ protein diets from weaning, given a single feed of ovalbumin or saline two weeks later, then the presence of tolerance assessed by comparing responses of ovalbumin and saline prefed animals to immunisation with ovalbumin in adjuvant. There were disparate effects on the humoral and cell mediated limbs of oral tolerance. Tolerance for serum antibody responses was more profound in protein deprived animals than in controls. Conversely, tolerance for DTH responses was impaired in protein deprived mice. Our results supported the hypothesis that protein deprivation selectively depletes the population of suppressor $T$ cells responsible for the fine control of DTH tolerance.

Impaired induction of oral tolerance for DTH responses to ovalbumin could also be explained by changes in the gut epithelium, with failure of the processing of fed antigen. This was studied with serum, collected one hour after feeding, which should contain tolerogenic, 'gut processed' antigen. ${ }^{10}$ Suppression of DTH was transferred with one hour serum from normal, protein sufficient mice, and also with serum from deprived mice, ${ }^{11}$ showing that their capacity for intestinal antigen processing was normal. Furthermore, the quantity of absorbed antigen in the serum one hour after feeding was similar in both protein deprived and normal groups.

\section{Adverse effects of elemental diet in infected mice}

We became interested in elemental diets and intestinal immunity when we first noted the beneficial effect of elemental diet treatment in Crohn's disease. ${ }^{12}{ }^{13} \mathrm{~A}$ series of experiments were performed in which mice were reared on an elemental diet (Vivonex standard), rendered solid by its incorporation in agar. A range of tests were used to examine humoral, cell mediated, and protective immunity. ${ }^{14}$ Interestingly, by comparison with mice fed on a normal diet the animals reared on an elemental diet were definitely less aggressive, they were lighter, their spleens and thymuses were smaller, and their peripheral white blood cell counts were lower. We noted that their livers were pale with histological examination showing a fatty infiltrate (presumably, because the constituents of the diet were not fully appropriate for nutrition of mice). Serum antibody responses to a protein antigen were impaired but cell mediated immunity, measured by the capacity to reject an allograft and mount delayed type hypersensitivity reactions of 
the skin, were normal. Studies of the gross anatomy of the mice clearly showed a tiny caecum in elemental diet mice when compared with normal diet animals, showing that bacterial flora and bacterial metabolism were probably substantially changed by this dietary manipulation.

In a separate series of studies experiments were carried out on the effects of elemental diet on mucosal architecture of mice by using conventional histological tests and a microdissection and stathmokinetic technique for measuring epithelial cell kinetics and tissue architecture. ${ }^{15}$ In uninfected mice, there were no differences in tissue architecture or in intraepithelial lymphocyte counts, when elemental diet and normal diet mice were compared. In a colony of mice with longterm infection with Giardia muris, however, there was a significantly greater increase in crypt length and metaphase accumulation in association with Giardia infection in elemental diet mice, than in Giardia infected normal diet mice when compared with normal diet controls.

Even more striking effects were seen in elemental diet mice infected with the nematode Nippostrongylus brasiliensis, with significantly reduced villus length and correspondingly raised crypt length and metaphase accumulation rate in mice on the seventh day of infection. Furthermore, there was evidence of composite immunodeficiency, reflected in an inability to clear worms from the gut in mice fed elemental diet, whereas worm counts dropped to near zero on the ninth day after infection in normal diet mice.

The reasons why a liquid or elemental diet might exacerbate tissue damage in the presence of an immune response to parasites are many. The stimulus for gastric acid will be changed, there are certainly profound changes in motility that would change the nature of the flora; the distribution of the gut flora along the length of the gut and in cross section is probably different in the absence of solid food matter; whether or not removal of normal food antigens is relevant is uncertain. It is conceivable that liquid or elemental diet actually enhances the metabolism of parasites and increases their virulence. All of these explanations would relate to a mechanism of increased mucosal damage by either increased parasite numbers, antigen quantity or virulence.

It is, however, equally possible that there is a change in immunoregulation at gut level in relation to unusual dietary constituents and that either mucosal antibody immunodeficiency, or up regulated inappropriate mucosal $\mathrm{T}$ cell function, is a direct effect of changed nutrition. If this were true, the findings of enhanced tissue damage in relation to parasites might also be duplicated in other situations of increased mucosal $\mathrm{T}$ cell function, for example cows' milk protein sensitive enteropathy, graft versus host reaction, allograft rejection. The fact that elemental diet is a useful treatment of so called unresponsive coeliac disease is reassuring in this context.

\section{Newly developed technique for clinical} investigation of gut mucosal immunity Research in nutrition is often carried out at the extremes of life, or in volunteers. In these situations invasive techniques for clinical investigation are often impossible or unethical. We have recently developed a method for obtaining intestinal perfusates by the use of whole gut lavage that overcomes this problem. ${ }^{16}$

Gut lavage is already widely used for bowel cleansing before barium enema, colonoscopy or colorectal surgery. In this procedure, patients drink several litres of isotonic non-absorbable fluid; this cleanses the gastrointestinal tract of all solid faecal material within two to three hours, after which virtually clear fluid, essentially a whole gut perfusate, is passed through the rectum. The fluid is processed by filtration and addition of a series of protease inhibitors. Aliquots of processed fluid are then stored at $-70^{\circ} \mathrm{C}$ for later analyses, for example by enzyme linked immunosorbent assay (ELISA).

We are using gut lavage tests to measure gastrointestinal protein and blood losses in inflammatory bowel disease and to investigate factors that regulate intestinal antibody biosynthesis and specific antibody production. Immunoregulatory and effector cytokines can also be detected in gut lavage fluid. This technique is commended as an excellent new approach to the clinical investigation of gut mucosal immunity, which can be exploited in many ways and would be ideal for research on nutritional rehabilitation and mucosal immunity.

1 Ferguson A, Parrott DMV. Growth and development of 'antigen-free' grafts of fetal mouse intestine. $\mathcal{F}$ Pathol 1972; 106: 95-101

2 Ferguson A, Parrott DMV. The effect of antigen deprivation on thymus-dependent and thymus-independent lymphocytes in the small intestine of the mouse. Clin Exp Immunol 1972; 12: 477-88.

3 Ferguson A, Gerskowitch VP, Russell RI. Pre- and postweaning disaccharidase patterns in isografts of fetal mouse weaning disaccharidase patterns in isografts

4 Parrott DMV, Ferguson A. Selective migration of lymphocytes within the mouse small intestine. Immunology 1974; 26: $571-88$

5 Ferguson A. Secretion of IgA into 'antigen-free' isografts of mouse small intestine. Clin Exp Immunol 1974; 17: 691-6.

6 Ferguson A, Parrott DMV. Histopathology and time course of rejection of allografts of mouse small intestine. Transplantation 1973; 15: 546-54.

7 MacDonald TT, Murray M, Ferguson A. Nippostrongylus brasiliensis: mast cell kinetics at small intestinal sites in infected rats. Exp Parasitol 1980; 49: 9-14.

8 Lamont AG, Gordon M, Ferguson A. T lymphocyte function in protein deprived mice. Clin Exp Immunol 1988; 72: 113-7.

Lamont AG, Gordon $M$, Ferguson A. Oral tolerance in protein-deprived mice. I. Profound antibody tolerance but impaired DTH tolerance after antigen feeding. Immunology 1987; 61: 333-7.

10 Strobel S, Mowat AMcI, Drummond HE, Pickering MG, Ferguson A. Immunological responses to fed protein antigens in mice. II. Oral tolerance for CMI is due to activation of cyclophosphamide-sensitive cells by gutprocessed antigen. Immunology 1983; 49: 451-6.

11 Lamont AG, Gordon M, Ferguson A. Oral tolerance in protein deprived mice. II. Evidence of normal 'gut processing' of ovalbumin, but suppressor cell deficiency, in deprived mice. Immunology 1987; 61: 339-43.

12 Ferguson A. Elemental diet: effects on immune status and on hypersensitivity reactions in the gut. In: Johnston IDA, ed. The role of elemental nutrition. Tunbridge Wells: MCS ed. The role of elemental nutrit

13 Logan RFA, Gillon J, Ferrington C, Ferguson A. Reduction of gastrointestinal protein loss by elemental diet in Crohn's disease of the small bowel. Gut 1981; 22: 383-7.

14 Ferguson A, Paul G, MacDonald TT. Immunodeficiency and fatty liver in mice reared on an elemental diet. Arq Gastroenterol 1978; 15: 11-5.

15 Ferguson A, Logan RFA, MacDonald TT. Increased mucosal damage during parasite infection in mice fed an elemental diet. Gut 1980; 21: 37-43.

16 O'Mahony S, Barton JR, Crichton S, Ferguson A. Appraisal of gut lavage in the study of intestinal humoral immunity. Gut 1990; 31: 1341-4. 